\title{
SIMULATION AND OPTIMIZATION OF POROUS BONE-LIKE MICROSTRUCTURES WITH SPECIFIC MECHANICAL PROPERTIES
}

\author{
Adrian Wit*, Sebastian Wronski, JACEK TARAsiuk
}

\author{
AGH University of Science and Technology, Faculty of Physics and Applied Computer Science, al. Mickiewicza \\ 30, 30-059 Kraków, Poland \\ * corresponding author: adrian.wit@fis.agh.edu.pl
}

\begin{abstract}
Bone trabecular structure can be characterized as a connected network of mineral bars and plates with unique mechanical properties. Standard methods of producing bone-like structures based on periodic structures or foams have same limitations. The organization of the trabecular bone (meso scale) is adapted to the values of stresses and strains affecting the skeletal system. To simulate bone-like structure, the methodology of generating stochastic structure based on hyperuniform spatial points distribution is proposed. Statistical analysis of generated structure shows the possibility to generate clouds of points in wide range of random close packing density, up to $59.52 \%$. Points connected by Voronoi tessellation produce to unique porous topology with no closed-cells and with wide range of connectivity. Manufacturing of a generated structure is only limited by used technique. The proposed algorithm was developed regardless of the manufacturing technique, however, same examples of the structure were printed using 3D addictive technology. The mechanical properties of developed structure are strongly dependent on the material from which they are made, but the modification of the structure allows to change the strength in specific and controlled way.
\end{abstract}

KEYWORDS: Bone modeling, Voronoi tessellation, porous structure.

\section{INTRODUCTION}

Simulating internal bones structure with proper mechanical behaviour is an extremely difficult task. Numerous study shows that the bioactive implants need specific requirements: high porosity and optimal pore size, directional strength and stiffness in range of natural bone, interconnected porous structure [1. The most often attempts to create trabecular structures based on repetitive nets composed of metallic or polymeric bars arranged on top of each other [2]. This periodic structures are characterized by isotropic mechanical properties and simple manufacturing process but they gave insufficient results in similarity to natural spongy bones structure. Another solutions use the methods of generating foam-like structures. However, the topology of standard foams leads to the formation of large areas of closed pores that do not allow free migration of living cells or the growth of blood vessels. The most realistic algorithm based on covering the space with 3-dimensional repetitive polyhedrons like Kelvin cells or quasicrystal polyhedrons as a results of a special decoration of Penrose tiling [3, 4]. They can form natural-looking net with roundly-shaped and connected porous. The scaffolds created on the edges of the polyhedrons and can be formed into various shapes and connections. The motivation to start working with designed structures was a significant progress in applications of 3D printed materials. Many studies present the possibility to print simple $3 \mathrm{D}$ porous structure, that can be successfully applied in the living organism [5, 6, however, they are far from obtaining a structure similar to natural bone. Researches on bone structure simulation are becoming more common but still they often have many limitations and simplifications [7]. The requirements of the good algorithm for generating base for bone-like structure are as follows: random spatial distribution, high uniformity points distribution which means that the points are not too close or too far to one another, no local concentration or grouping tendency and no preferred direction of distribution. The proposed methodology of generating porous structure is based on stochastic algorithm with well-spaced points distributions and real-time generation. Very important role in the generator plays the real-time processing which allow to build adaptive algorithm targeted to reproduce the bone-like implants based on medical images.

\section{MATERIALS AND METHODS}

The basis of the model is blue-noise spatial points generator. The well-spaced blue-noise combine two desirable properties: random distribution and uniform space coverage. The modification of Bridson fast sampling method [8] allow to easily generate large amount of points in any limited space. It also ensure the great homogeneous points distribution in local volume as well as good repeatability of generations with the satisfactory minimal margin of error. The set of points are connected together by using Voronoi tessellation. Postprocessing of the structure allow us 
to modify the shape and size of structure scaffolds.

\subsection{Spatial points ClOUd generator}

Let's initialize an limited 3-dimensional background space with size $s_{\mathrm{x}}, s_{\mathrm{y}}, s_{\mathrm{z}}$ and starting seed with coordination's: $x_{0}, y_{0}, z_{0}$. Then create the list of active points that contains points in which domain it is possibility to generate next points, and conflict list for checking if the newly generated point is not located too closely the others. Next step is to randomly generate a new seed that meets conditions: $x_{\mathrm{n}}, y_{\mathrm{n}}, z_{\mathrm{n}}>r_{\mathrm{f}}$ and $x_{\mathrm{n}}, y_{\mathrm{n}}, z_{\mathrm{n}}<r_{\mathrm{c}}$. The space between $r_{\mathrm{c}}-r_{\mathrm{f}}$ is called a point domain, where new points will be generated. While the list of active points is not empty, continue generating additional points and check if there are not too close to other points. If the conditions about location are correct, save this points in list of active points and continue the generation. In respect of each points, algorithm try to generate k-points in the domain. While $\mathrm{k}$ number is reached, remove the seed from the active list and randomly choose another seed from the list. In Table 1, they have been collected all the initial parameters for the generator.

The generator continue while the list of active points is not empty. The generator cannot allow to set new points out of limited space, so the number of points is numerally finite. The size of the background, $\beta$-ratio, and $k$-hits affects the number of points and density of packing. The graphical interpretation of points generation process and final results of spatial points cloud generator are presented in Figure 1

The value of $\beta$-ratio was tested in wide range between 1 and 100, depending on the planned density of the structure and the connectivity of scaffolds. The value of $k$-number was set between 1 and 2000, but optimal value was defined at about 50. Higher values heavily extends the time of calculations. Both parameters should be tested individually and settings depending on the type or size of structure generated and assumption of implant porosity and the 3D-printer available parameters.

\subsection{VORONOI-BASED STRUCTURE}

Voronoi diagrams are related to one of the most fundamental constructs defined by a discrete set of points. The aim of the Voronoi is to automatically build a trabecular structure based on a set of points in the limited volume. The result of this operation is a group of adherent tetrahedrons connected into 3D network with the desired hyperuniform properties. Cells obtained in this way are mostly regular and similar with sizes (expect connected with borders). The results of Voronoi diagram based on 3D well-placed sets of points is present in Figure 2

The output Voronoi diagram was processed into series of rod using Grasshopper, the graphical editor integrated into Rhinoceros 5. Trabeculae were built
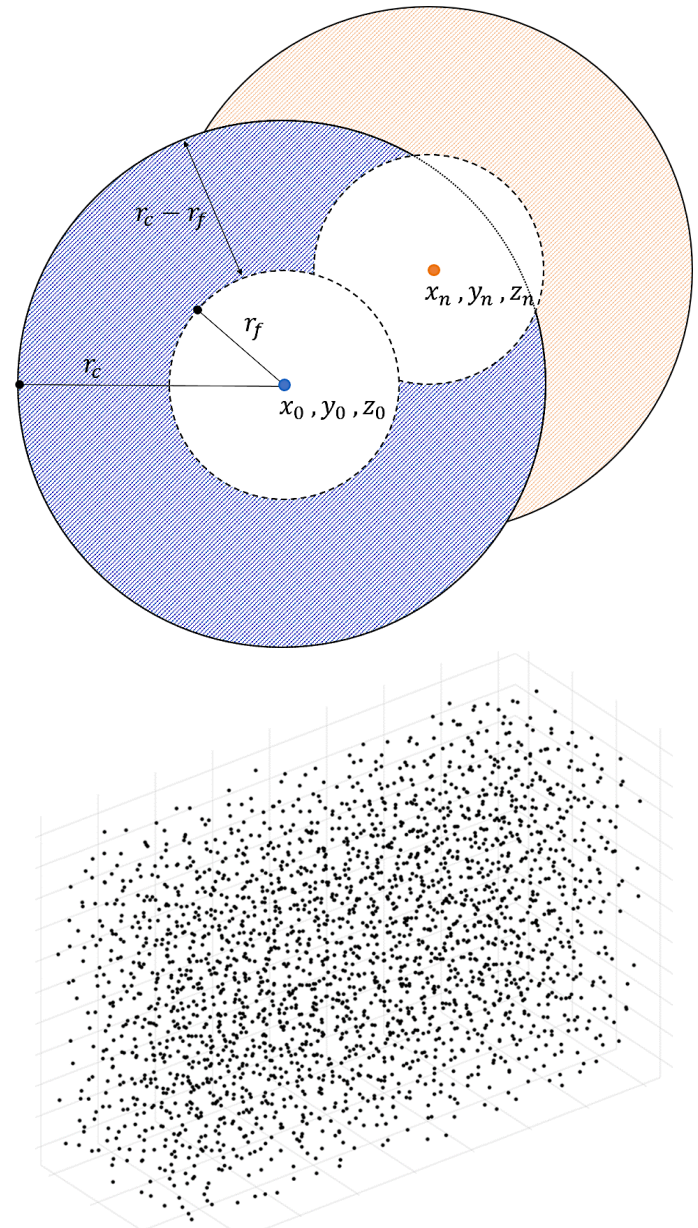

FiguRE 1. Illustration of initial parameters during domain filling. No more points can exist in white circle (upper image). Results of filling the 3-dimensional space with uniform points distributions (lower image).

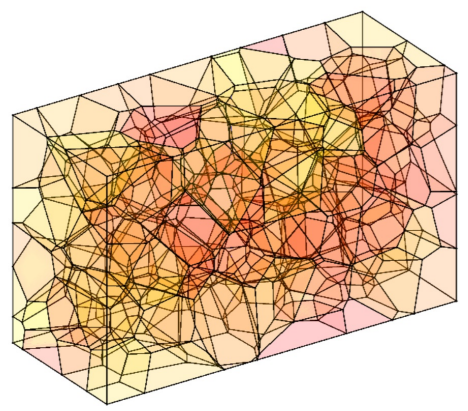

Figure 2. An example of using 3D Voronoi diagram based on hyperuniform points cloud.

on the tetrahedrons edges achieved from Voronoi. In Grasshopper, rods can be designed with the various shapes, sizes and connection. All these parameters affect the final parameters of the model. Changing the thickness of the trabeculae causes a change in the porosity of the structure and further the mechanical properties. The final result of the Grasshopper is a meshed model that has been saved in Standard 


\begin{tabular}{ccc}
\hline $\begin{array}{c}\text { Symbol } \\
s_{\mathrm{x}}, s_{\mathrm{y}}, s_{\mathrm{z}}\end{array}$ & Name & $\begin{array}{c}\text { Description } \\
\text { Background size }\end{array}$ \\
\hline$x_{0}, y_{0}, z_{0}$ & Initial seed coordination's & coordination's of initial seed inside the background. \\
& This point could be generated randomly or arbitraly \\
$r_{\mathrm{f}}$ & Conflict radius & the minimum distance between any pairs of points \\
$r_{\mathrm{c}}$ & Coverage radius & the maximum distance between any pairs of points \\
$k$ & Hits limit & the maximum number of attempts to fill a points domain \\
$\beta$ & $\beta$-ratio & the ratio between the radius of the conflict
\end{tabular}

TABLE 1. List of initial parameters for spatial points cloud generator

Triangle Language (STL) format which is ready for printing.

\subsection{AdDictive manufacturing}

The final stage of the work is to produce bone-like porous structures. For manufacturing the artificially porous models in macro-scale the modified B9Creator DLP printer was used. Digital Light Processing (DLP) uses a liquid photopolymer resin which is able to be solidify under a light source with specific wavelength (typically in the range of UV light). In DLP printer, the light source project a single slice at the bottom of the vat, which causes the liquid resin cure in specific pattern. The solidified resin stick to the table and are removed from the resin so the next layer can be lightening. This printer is characterised by printing detail at $30 \mu \mathrm{m}$ microns resolution and maximal model size $104 \times 75 \times 203 \mathrm{~mm}$. However, our modification of the printer allow to print trabecular structure with minimums size of element less than $20 \mu \mathrm{m}$. This is a sufficient resolution to reproduce the size of the natural bone trabeculae existing in the human bone. The mechanical properties of the printed sample strongly depend on the strength properties of the resin. The proposed methodology for creating structures is independent from the printing material, so the final structure strength was not measured. Depending on the manufacturer, the properties of the resins can be significantly different. There are many materials with very high stiff, chemical resistance and biocompatibility.

\section{Results}

The distribution of different sets of points have been analysed and measured. The most important properties defined for isotropic distribution were global and local density. Global density was measured as a sum of all spheres limited by conflict radius dividend by the volume of the entire space. Total density for different $\beta$-ratio and $\mathrm{k}$ parameter were measured and presented at Figure 3 Increasing the number of $k$-hits strongly influence the global density of the points distribution. The local density was measured by constantly dividing the background for small (equal in volume) sectors and measure the average number of the points in every
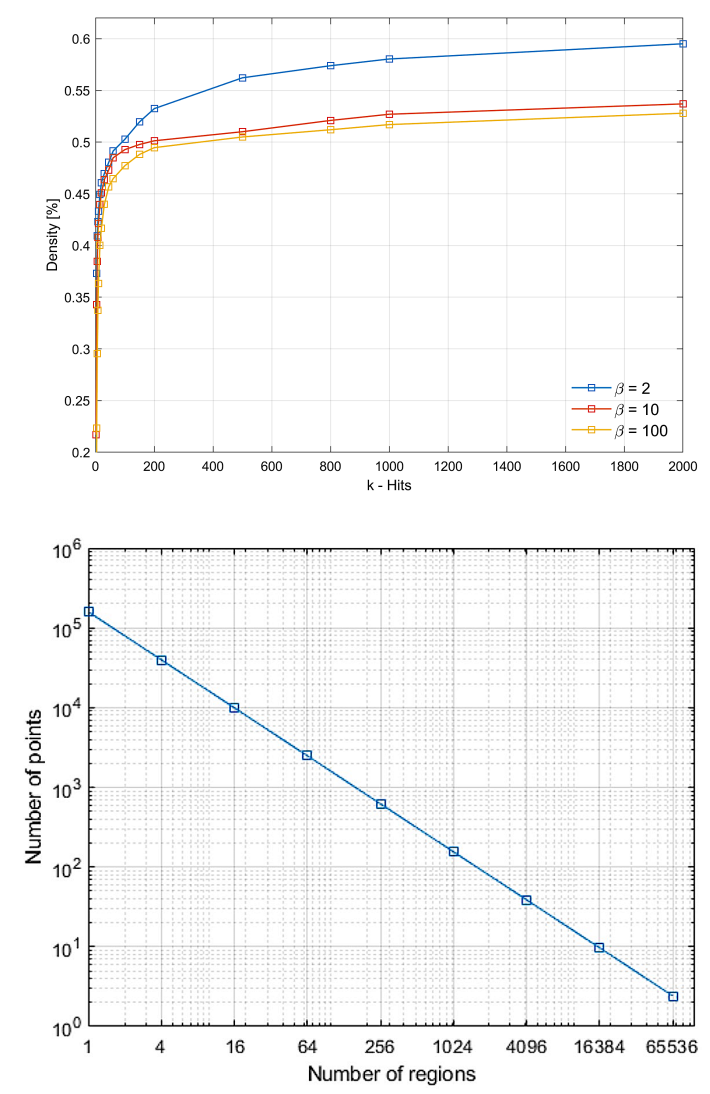

Figure 3. The density of points in function of $k$-hits show the increasing packing density with increasing $\mathrm{k}$ number (upper image). The average numbers of points in different number of squares (lower image).

sector. The standard deviation between the number of points in each volume was calculated. For the sample that contains 176,531 points, when divided into 65536 regions, the every sector contains proximity 2.81 of points with STD $=0.056$.

For the best parameters with high $\mathrm{k}$ (more than 2000) parameter and low $\beta$-ratio (less than 2) we are able to achieve the close packing density of about $59.52 \%$. The largest theoretical packing density has a crystalline facecentered cubic structure (FCC) which is $74.048 \%$. The best packing methods using random distributions allow obtaining results between $60-65 \%$. The appropriate 

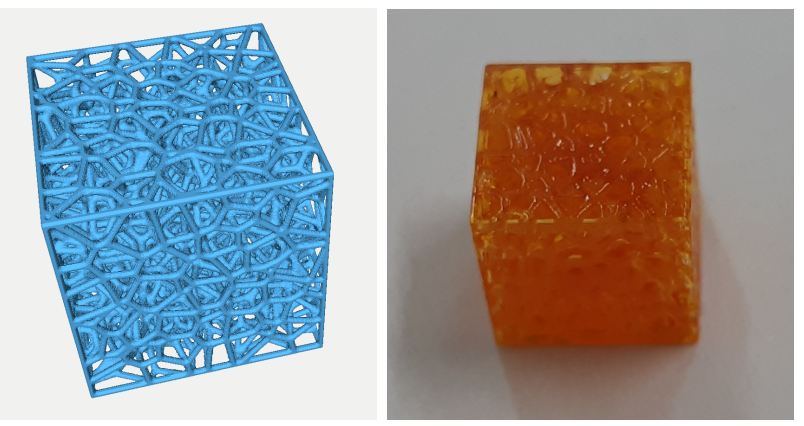

FigURE 4. The numerical model of porous structure prepared for the addictive manufacturing (left). The printed model using DLP technology. The size of the printed cube is $10 \times 10 \times 10 \mathrm{~mm}$ (right).

model of our structure has been prepared and printed using DLP technique. The result was shown in Figure 4 .

However, the printing was developed only for visual inspection. As the main goal of this work was to create methodology for trabecular structure designing, the mechanical properties of the structure and biocompatibility were not analysed. The simplest geometrical parameters like a porosity, trabecular thickness, number of scaffolds were not measured and will be continued in the upcoming works. The biocompatibility of the resin was not defined. The final mechanical properties of the structure was not yet measured.

\section{Conclusion}

The method of generating bone-like structure based on blue noise algorithm give promises results in the matter of equal distribution of points and wide range of density obtained. The minimal and maximal value of archived density by the method is very important for designing bones with a significant variation of density, from cortex bones to porous bone fragments. Additionally the generator is characterized by a good repeatability in the satisfactory minimal size of structures. The methods of generating points cloud disturbing can be improved. Several studies show the numerical methods to improve spatial distraining with postprocessing algorithms [9]. Using Voronoi diagram to create open and connected cell gives satisfactory results.

The purpose of further work will be improving the shape of the cell to be more roundish and increase their space diversity. On the other hand, before continue of this work a correct biocompatible resin has to be chosen, when work on printing and testing the mechanical properties of the samples will be continued. The method requires developing a set of optimal parameters like exposure time and time of disappearance for a sample of different sizes and porosities. Also the compression test to analyse the mechanical properties are planned.

\section{ACKNOWLEDGEMENTS}

This work was financed by the Polish National Centre for Science (NCN) under decision number: 2017/26/E/ST5/00043. Adrian Wit has been partly supported by the EU Project POWR.03.02.00-00-I004/16.

\section{REFERENCES}

[1] J. Teo, K. Si-Hoe, S. Teoh. Relationship between ct intensity, micro-architecture and mechanical properties of porcine vertebral cancellous bone. Clinical

Biomechanics 21:235-244, 2006. DOI:10.1016/j.clinbiomech.2005.11.001

[2] B. Thavornyutikarn, N. Chantarapanich, K. Sitthiseripratip, et al. Bone tissue engineering scaffolding: computer-aided scaffolding techniques. Progress in biomaterials 3:61-102, 2014. DOI:10.1007/s40204-014-0026-7.

[3] W. Jang, A. Kraynik, S. Kyriakides. On the microstructure of open-cell foams and its effect on elastic properties. International Journal of Solids and Structures 45:1845-1875, 2008. DOI:10.1016/j.ijsolstr.2007.10.008.

[4] M. Sniechowski, J. Kaminski, S. Wronski, et al. Heterogeneous materials based on aperiodic structures for bone tissue substitutes. In VI International Conference on Computational Bioengineering. 2015. https://www.researchgate.net/publication/ 313040001_HETEROGENEOUS_MATERIALS_BASED_ON APERIODIC_STRUCTURES_FOR_BONE_TISSUE_ SUBSTITUTES

[5] A. Palmquist, F. Shah, L. Emanuelsson, et al. A technique for evaluating bone ingrowth into $3 \mathrm{~d}$ printed, porous ti6al4v implants accurately using $\mathrm{x}$-ray micro-computed tomography and histomorphometry. pp. 1-8, 2017. DOI:10.1016/j.micron.2016.11.009

[6] J. Megias-Barrera, L. Garcia-Consuegra, A. Novoa, et al. Histological and radiological evaluation of subcutaneous implants in mouse of a $3 \mathrm{~d}$-printable material (fulcure 720) and experimental application in mandibular reconstruction. pp. 88-92, 2018. DOI:10.1016/j.jormas.2017.11.004

[7] M. Fantini, M. Curto, F. de Crescenzio. A method to design biomimetic scaffolds for bone tissue engineering based on voronoi lattices. Virtual and Physical

Prototyping 11(2):77-90, 2016. DOI:10.1080/17452759.2016.1172301.

[8] R. Bridson. Fast poisson disk sampling in arbitrary dimensions. ACM SIGGRAPH 2007. DOI: $10.1145 / 1278780.1278807$

[9] M. Ebeida, M. Awad, X. Ge, et al. Improving spatial coverage while preserving the blue noise of point sets. Computer-Aided Design 46:25-36, 2014. DOI:10.1016/j.cad.2013.08.015 